\title{
CYTOGENETIC STUDIES OF RECIPROCAL TRANSLOCATIONS INDUCED BY X-RAYS IN TRADESCANTIA PALUDOSA*
}

\author{
HIROSHI WATANABE \\ Department of Biology, Faculty of Science, Kyushu University, Fukuoka 812, Japan
}

Received 21.viii. 72

\begin{abstract}
SUMMARY
In Tradescantia paludosa $(2 n=12,6 \mathrm{II})$, by X-raying resting axillary buds at 10,000 or $5000 \mathrm{R} ., 43$ primary strains heterozygous for reciprocal translocations were reared vegetatively by cuttings. Some of the primary strains were irradiated again in the same way, and nine secondary strains involving higher classes of chromosome catenation were reared to maturity. The ultimate strain $\odot 12$ was obtained through a third irradiation of a secondary strain $2 \odot 6$ which derived from a primary strain $\odot 6$. Absence of branches chimerical for different chromosome constitutions suggested that the new branches from the irradiated buds have developed from a single primordial cell. Theoretically, in longlived perennials reciprocal translocations can accumulate vegetatively by successive interchanges without crossing or interbreeding the translocation strains. The accumulation culminates in formation of $\operatorname{strain}(s)$ of ring-of- $2 n$. This was proved to be possible by the present study.
\end{abstract}

\section{INTRODUGTION}

IN certain perennial plants, meiotic rings consisting of all members of the zygotic chromosome complement are known, e.g. $\odot 14$ in Oenothera $(2 n=14)$, $\odot 10$ in Paeonia $(2 n=10)$ and $\odot 12$ in Rhoeo $(2 n=12)$ (cf. Burnham, 1956). Evolutionary processes which lead to the formation of the ultimate rings in these perennial plants have been discussed by several workers. Their conclusions may be summarised in the following three categories: (1) the gradual accumulation of reciprocal translocations by a plant until all chromosomes are involved in a ring (Darlington, 1931), (2) intercrossing between different reciprocal translocation strains (Cleland, 1962), and (3) co-operation of both of these two processes (1) and (2). Experimentally, Yamashita (1951, 1952, 1953) confirmed the process (2) by synthesising an ultimate $\odot 14$ strain in Einkorn wheat $(2 n=14)$ through hybridisation between different X-ray induced $\odot 4$ strains.

Since the rings composed of large numbers of chromosomes cause striking gametic abortion, propagation of the heterozygotes with a large number of translocations is reduced greatly in sexual annual plants unless they are counteracted by some mechanisms such as a balanced lethal system, as in Oenothera (Cleland, 1962), genetic control for alternative segregation as in Secale (Thompson, 1956; Lawrence, 1958), and so forth. On the other hand, in perennial plants which propagate vegetatively, the rings composed of large numbers of chromosomes are easily maintained asexually. Therefore, they are able to accumulate translocations successively until they form an ultimate ring of zygotic complement.

- Contributions from the Department of Biology, Faculty of Science, Kyushu University, No. 185. 
The purpose of the present study is to present in detail an experimental confirmation of the process (1), i.e. successive accumulation of the X-ray induced reciprocal translocations, from $6_{\text {II }}$ to a $\odot 12$ in Tradescantia paludosa $\left(2 n=12,6_{11}\right)$, a perennial plant. The results of this investigation were partly reported in previous papers (Watanabe, 1959, 1962a,b).

\section{Materials and MEthods}

A progeny of a stock Bussey No. 5 of Tradescantia paludosa Anderson and Woodson $(2 n=12+1 \sim 12 f)$ was used exclusively in the present study.

Cuttings with two or three resting axillary buds were removed from all branches of the mother plant, with or without roots. The buds were irradiated at 10,000 R., a single lot among the plants irradiated for the first time, which was irradiated at $5000 \mathrm{R}$; By observing the meiosis in PMCs formed on newly developed branches from the irradiated resting axillary buds, branches heterozygous for reciprocal translocation were selected out and propagated vegetatively through cuttings. Cuttings from some of these primary strains were irradiated for the second time in the same way as the first irradiation, and strains more complicated than the primary strains were obtained. Of these secondary strains, some were irradiated for the third time in the same way as the preceding irradiations.

Meiotic configurations were observed at MI in PMCs fixed in a solution containing propionic acid and smeared in aceto-carmine (Newcomer, 1953).

To observe somatic mitosis, root tips were pretreated in $0.002 \mathrm{M} 8$ oxyquinoline for 2-4 hours, and/or axillary bud meristems were fixed in La Cour 2BE, and squashed after Feulgen-staining.

\section{TransLocations}

Cells in mitosis are found in primordial tissues of the axillary buds a week or more after the cuttings. Mitoses in the irradiated buds revealed aberrations such as breakage of metaphase chromosomes, bridges and fragments at anaphase, micronuclei at interphase, etc. (plate I, 1 and 2). In the controls, however, none of these aberrations was found (plate I, 3). Irregularities observed in $\mathrm{X}$-rayed cells suggest that many breakages and reunions of chromosomes were induced in resting nuclei by the irradiation.

In spite of induced chromosome aberrations and mitotic irregularities, the new branches developed from X-rayed resting buds were revealed to consist of only a single chromosome constitution, the standard, or certain single constitutions heterozygous for a translocation or translocations. None of the branches were chimerical for the different constitutions of the chromosomes. Therefore, it seems reasonable to conclude that the new branches from the irradiated buds usually result from a single cell which is genetically well balanced, but may or may not comprise translocations. The same conclusion was stated in the case of barley spikes developed from irradiated dormant seeds (Caldecott and Smith, 1952).

The standard strain normally forms six bivalents at MI (plate II, 4). $\mathrm{X}$-irradiations of the standard strain yielded many different strains of translocations, which took place between two non-homologous chromosomes, between more than two chromosomes, and so forth. The first irradiation yielded 43 primary strains heterozygous for reciprocal translocations (table 1). 


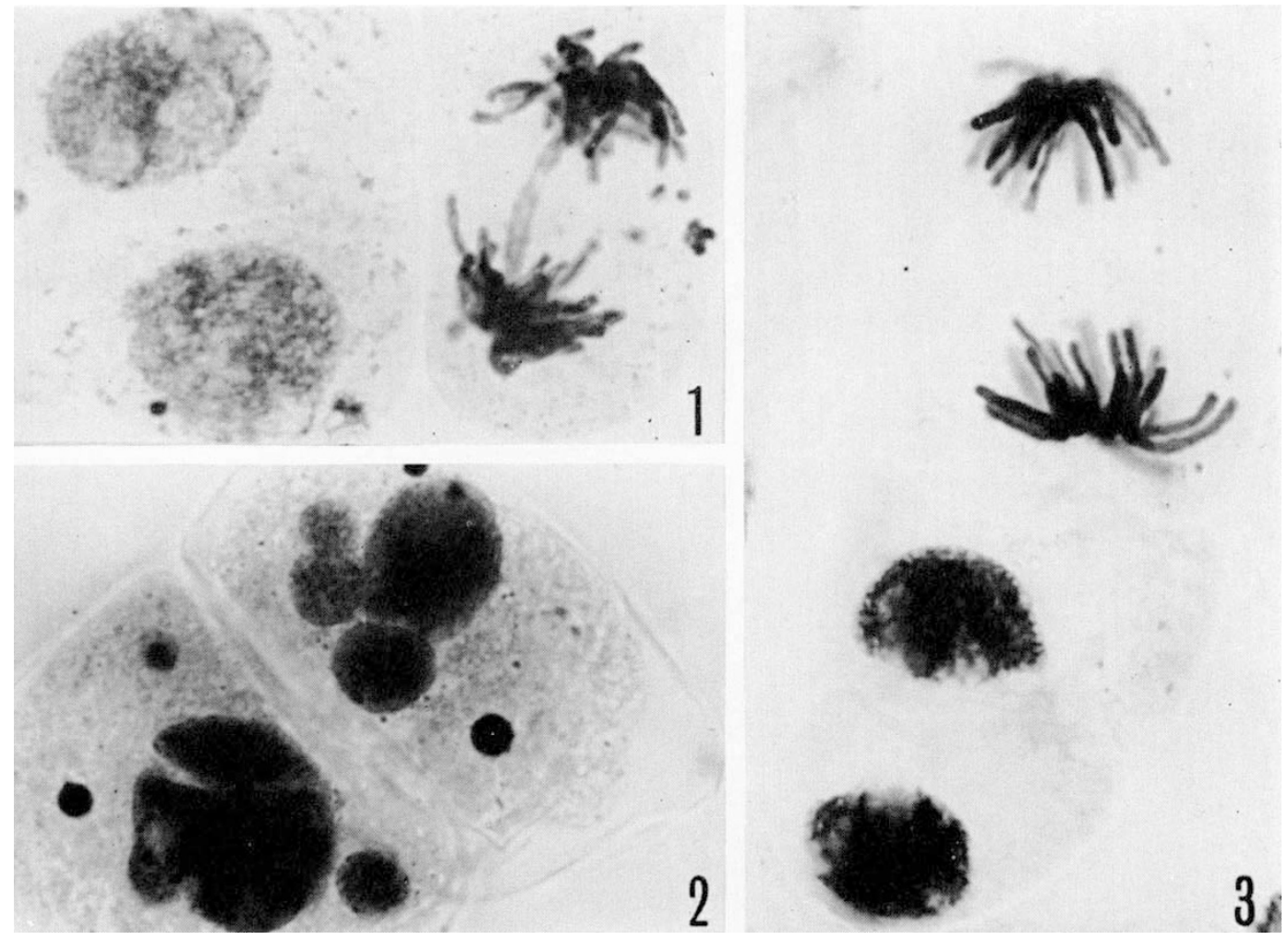

Plate I

Mitoses in axillary bud meristem. $\times 1500.1$ and 2, Bridges, fragments and micronuclei in the cells dividing after $\mathrm{X}$-irradiation. 3, Dividing cells showing no aberrations in a control plant. 

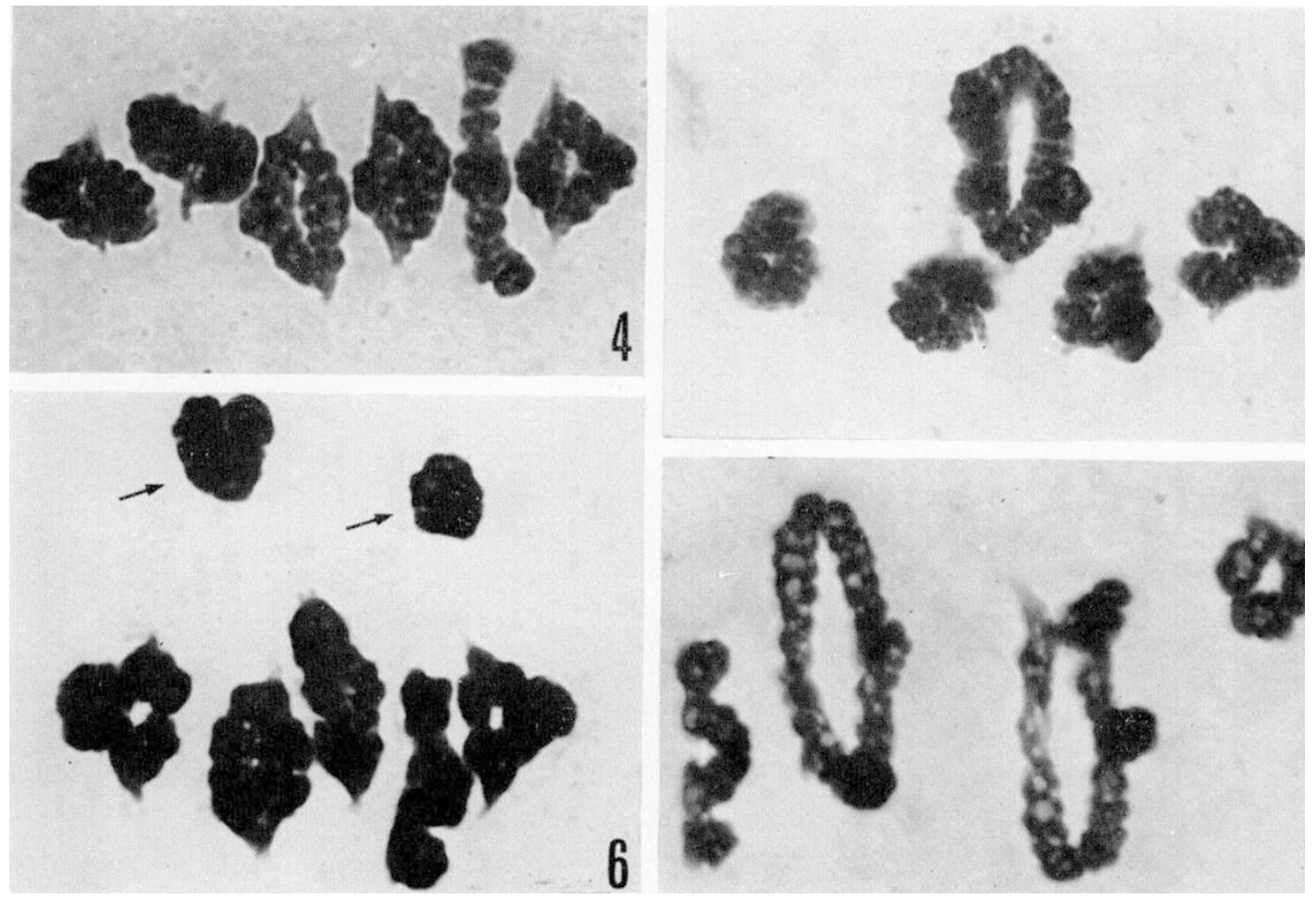

Plate II

MI configurations in PMCs of X-ray induced primary strain. $\times 1500.4,6$ II, standard strain. $5, \odot 4+4_{\mathrm{II}} \cdot 6,2 \odot 1+5_{\mathrm{II}}$, arrow indicates $\odot 1.7,2 \odot 4+2_{\mathrm{II}}$. 

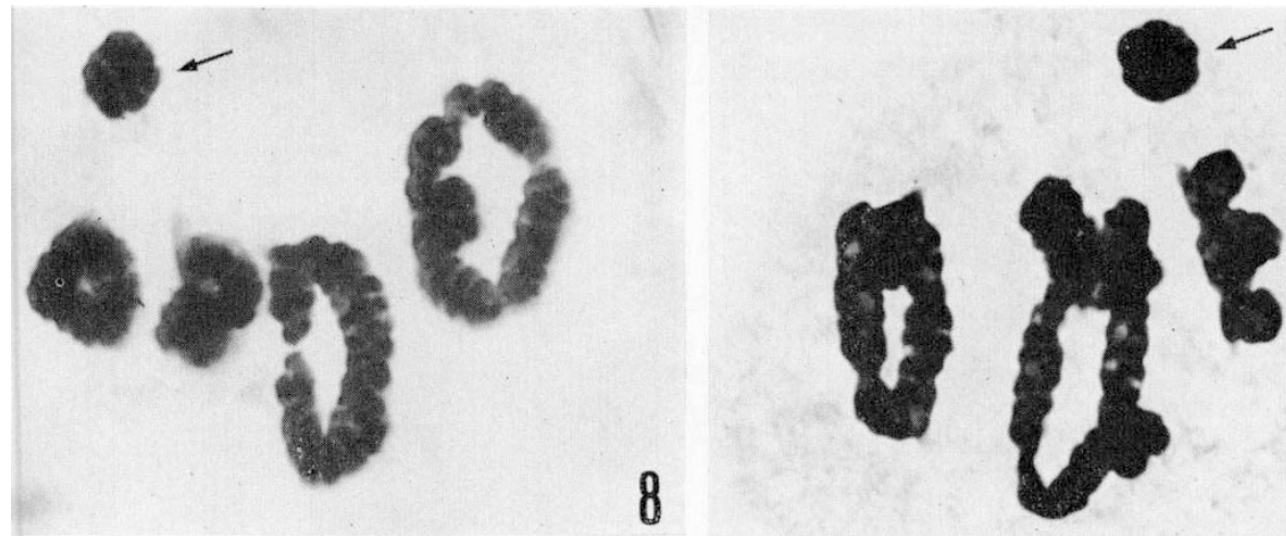

9

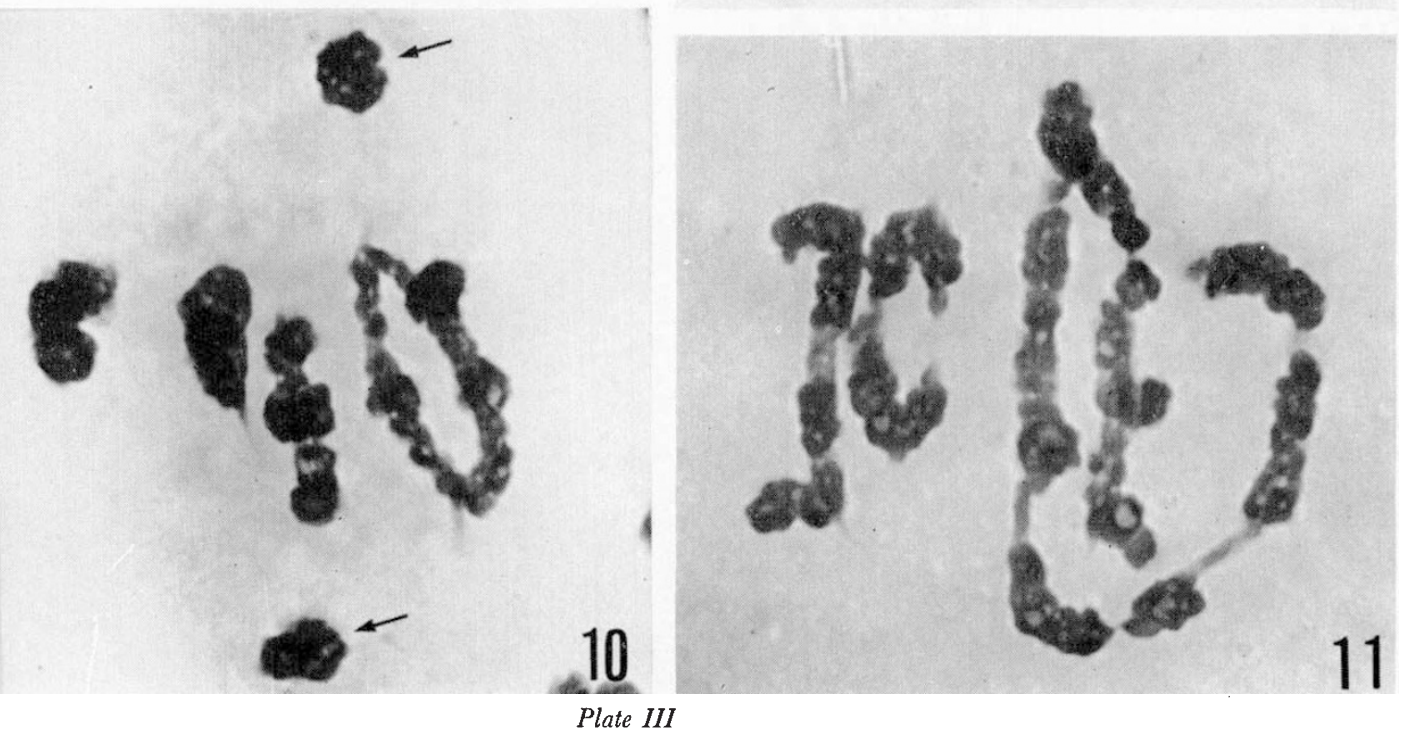

MI configurations in PMCs of the X-ray induced strains. Arrow indicates $\odot 1, \times 1500$. $8, \odot 4+\odot 3+\odot 1+2_{\mathrm{II}}$, primary strain from the standard. $9, \odot 6+\odot 3+\odot 1+1_{\mathrm{II}}$, secondary strain from primary strain $\odot 4+\odot 3+\odot 1+2_{11}(8) .10, \odot 4+2 \odot 1+3_{11}$, primary strain from the standard. $11, \odot 8+2_{I I}$, secondary strain from primary $\odot 4+2 \odot 1+3_{11}(10)$. 

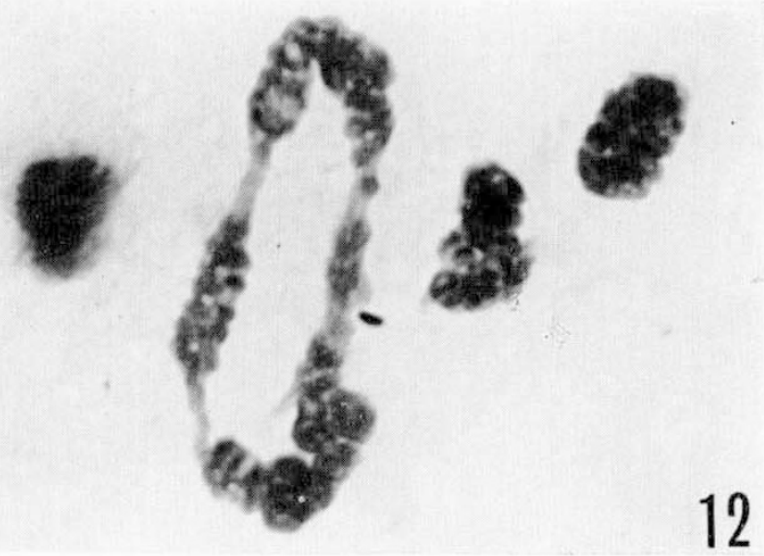

12
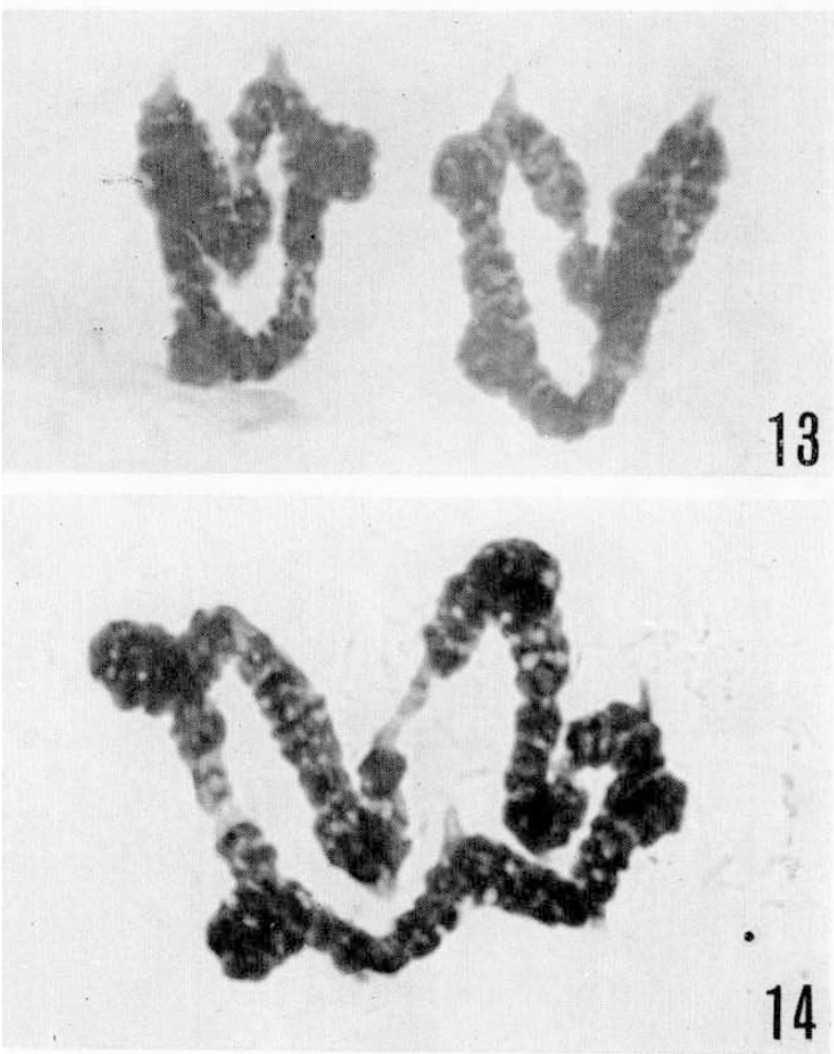

MI configurations in PMCs of X-ray induced strains showing three steps to $\odot 12$ from $6_{\text {II }}$. $\times 1500.12, \odot 6+3_{1 \mathrm{I}}$, a primary strain. $13,2 \odot 6$, a single secondary from a primary strain $\odot 6+3_{\text {II }}(12)$. 14, $\odot 12$, a single tertiary strain from the secondary $2 \odot 6(13)$. 
The most frequent type of reciprocal translocation detected was $\odot 4+4_{\text {II }}$, a simple reciprocal translocation between non-homologous chromosomes, 31 strains (plate II, 5). Two strains showed $2 \odot 1+5$ II, a reciprocal translocation between non-homologous arms of a homologous chromosome pair. Two doughnut-shaped pseudo-isochromosomes, both of which were smaller than any of the bivalent, showed no association with each other or with any other chromosomes (plate II, 6). The other strains, $2 \odot 4+2$ II (plate II, 7), $\odot 6+3_{\text {II }}$ (plate IV, 12), $\odot 4+\odot 3+\odot 1+2$ II (plate III, 8) and $\odot 4+2 \odot 1$ +3 II (plate III, 10), are those complicated a little more than the preceding cases, resulting from three or more breakages and reunions.

From irradiating resting axillary buds of the primary strains, nine more advanced secondary strains were obtained, e.g. $\odot 6+3_{\text {II }}$ and $2 \odot 4+2$ II from the primary $\odot 4+4$ II strains. Of these $\odot 6+3_{I I}$ is a result of a secondary addition of a reciprocal translocation which involved a member of the

TABLE 1

Meiotic pairing in $X$-ray-induced translocation strains*

\begin{tabular}{|c|c|c|c|c|}
\hline \multicolumn{2}{|l|}{ Primary strains } & \multicolumn{2}{|l|}{ Secondary strains } & Tertiary strain \\
\hline$\odot 4+4 I I$ & $(31)$ & $\begin{array}{l}\odot 6+3_{11} \\
2 \odot 4+211\end{array}$ & $\begin{array}{l}\text { (1) } \\
\text { (1) }\end{array}$ & - \\
\hline $2 \odot 1+5_{I I}$ & (2) & - & & - \\
\hline$\odot 6+3_{I I}$ & (4) & $\begin{array}{l}2 \odot 6 \\
\odot 6+\odot 4+1 I I\end{array}$ & $\begin{array}{l}\text { (1) } \\
\text { (1) }\end{array}$ & $\stackrel{\odot 12}{-}$ \\
\hline $2 \odot 4+2 I I$ & (3) & $\begin{array}{l}\odot 8+211 \\
\odot 6+\odot 4+11\end{array}$ & (1) & - \\
\hline$\odot 4+2 \odot 1+3 \mathrm{II}$ & (1) & $\odot 8+2 n$ & (1) & - \\
\hline$\odot 4+\odot 3+\odot 1+2 \mathrm{II}$ & $\begin{array}{r}(2) \\
(43)\end{array}$ & $\begin{array}{l}\odot 5+\odot 4+\odot 1+1_{I I} \\
\odot 6+\odot 3+\odot 1+1_{I I}\end{array}$ & $\begin{array}{l}\text { (1) } \\
(1) \\
\text { (9) }\end{array}$ & - \\
\hline
\end{tabular}

* "๑ " means " ring-of-" or " chain-of-". Numbers of strain induced are given in parentheses.

primary $\odot 4$, and $2 \odot 4+2$ II result of a translocation between a nonhomologous pair of chromosomes which has not participated in the primary $\odot 4$.

Among a family of the third irradiation of the second strain $2 \odot 6$ (plate IV, 13 ), a secondary strain from a primary strain $\odot 6+3_{\text {II }}$ (plate IV, 12), a plant with an ultimate ring $\odot 12$ was obtained (plate IV, 14).

In somatic mitoses, six chromosome pairs of the standard strain are classifiable into two groups by their centromere position. Three pairs are non-satellited with median or nearly median centromeres, and the remaining three pairs, with sub-median centromers, have minute satellites at their short arm ends (fig. 15). Based on these karyotypic characteristics, in certain favourable cases it was possible to identify the chromosome arms which were involved in reciprocal translocations. Thus, a primary strain of $\odot 4+4$ II was deduced to have involved a reciprocal translocation between a satellited short arm of one of the three sub-median chromosomes and an arm of three non-satellited median chromosomes (fig. 16). In this case a satellite made the identification reliable despite the nearly equal size of the interchanged segments. In another primary strain of $\odot 6+3$ II, on the other hand, marked morphological alterations were found in three chromosomes (fig. 17). This circumstance suggests a result of three reciprocal translocations involving interchanged segments of markedly different sizes. Of course, the same 

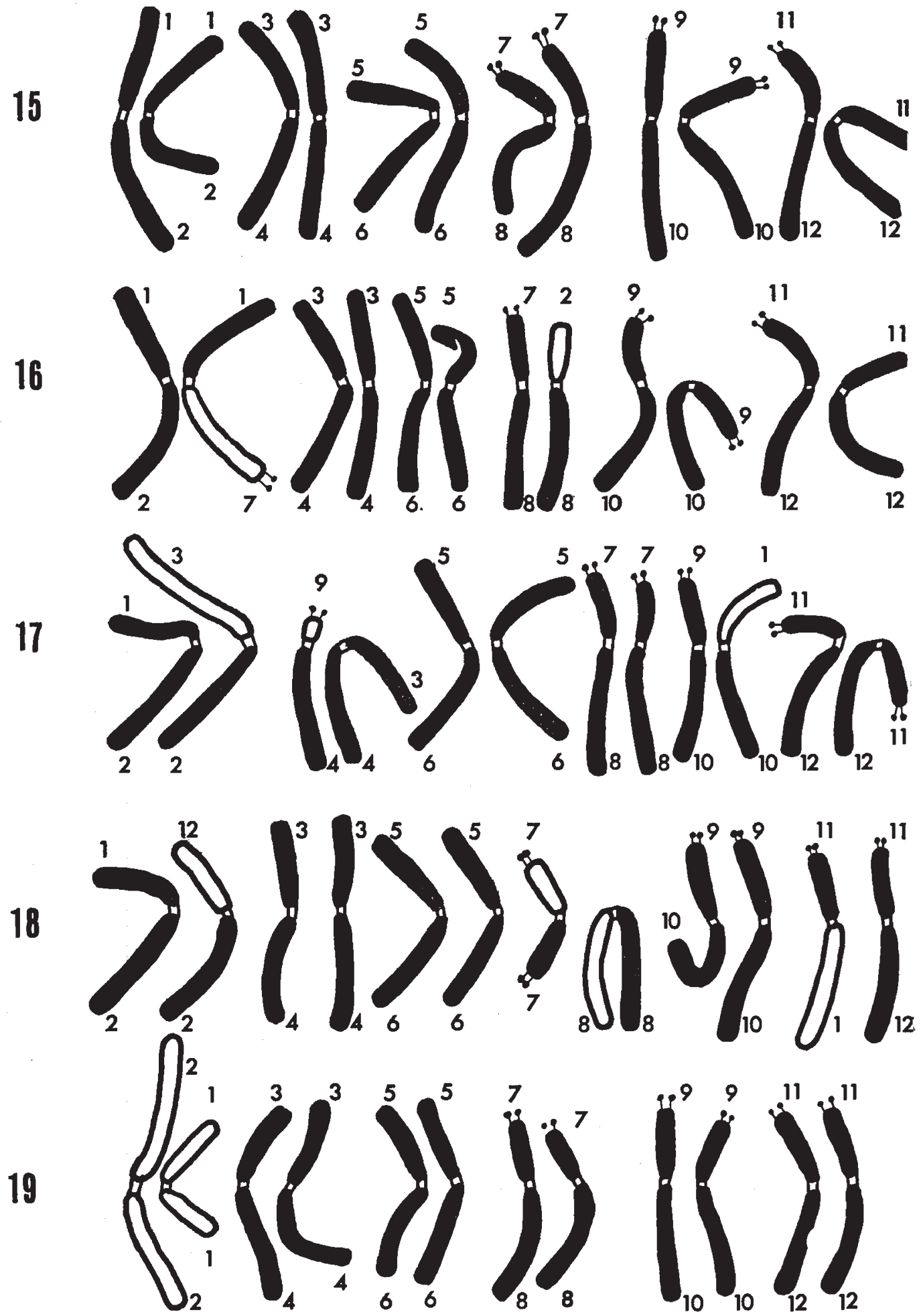

Fros. 15-19.-Chromosome sets in root-tip cells of standard and X-ray induced translocation strains. Unaltered arms are shown in black and altered ones in blank. $\times 2000$.

Fro. 15. - $\mathrm{III}_{\mathrm{I}}$, standard strain. Homologous arms are indicated with the same numerals; 1 to 12 .

Fro. 16. $-\odot 4+4$ II, primary strain. 1.7 and 2.8 are the interchanged chromosomes.

Fic. 17. $-\odot 6+3_{\text {II }}, 2 \cdot 3,4 \cdot 9$ and $1 \cdot 10$ are interchanged chromosomes.

Fig. 18.- $\odot 4+2 \odot 1+3_{\mathrm{II}}, 7 \cdot 7$ and $8 \cdot 8$ are pseudo-isochromosomes.

Fic. 19. $-2 \odot 1+5$ II, $1 \cdot 1$ and $2 \cdot 2$ are pseudo-isochromosomes. 
numerals in different figures do not imply genetic identity, which can not be established without crossing experiments.

A primary strain which showed a configuration $\odot 4+2 \odot 1+3$ II (plate III, 10), revealed two isobrachial chromosomes in somatic mitoses. One of the two was the longest and the other the shortest chromosome attached by a satellite at its both terminal ends (fig. 18). Two pseudo-isochromosomes found in a primary strain $2 \odot 1+5$ II (plate I, 6) were isobrachial and of markedly different lengths not to be expected as a doubled short and doubled long arm length (fig. 19). This case might have resulted from more than two break-and-reunions. A case of special interest is found in one secondary strain, in which two pseudo-isochromosomes reverted into two chromosomes each with ends of different segments, i.e. reversion from $\odot 4+2 \odot 1+3$ II (plate III, 10) to $\odot 8+2$ II (plate III, 11).

Generally speaking, the second irradiation of the primary strains induced the addition of new rings and/or enlarged the ring by a new translocation between a member of its primary ring and a member remained intact at the first irradiation. None of the secondary strains showed reduction of the number of translocated chromosomes (table 1). Rapid enlargement of a ring will result by a translocation between members of two independent primary rings, e.g. a secondary strain $\odot 8+2$ II from a primary strain $2 \odot 4+2 \mathrm{II}$. Indeed, a spectacular case of formation of an ultimate $\odot 12$ by only three steps was recorded in the present study as described below. The first irradiation yielded a primary strain $\odot 6+3$ II (plate IV, 12; fig. 20). Irradiating this strain I obtained a secondary strain $2 \odot 6$ (plate IV, 13; fig. 21). Finally, the third irradiation of this strain realized a joining $2 \odot 6$ into an ultimate ring-of-twelve (plate IV, 14; fig. 22).

The processes of originating to this strain $\odot 12$ is very simple and is of great evolutionary significance, since alternate segregation of the ring results in normal and complete gametic sets without duplication and/or deficiency of any segment of chromocomes.

\section{Fertility}

Pollen fertility was estimated by counting pollen grains well stained with aceto-carmine. The fertility was reduced with increased number of reciprocal translocations. Mean fertility in percentage was 70.5 in the strains with $\odot 4$, an estimate in the standard strain being $90 \cdot 3$, i.e. 20 per cent. decrease. Nearly the same situation, 20 per cent. decrease, was seen in the strains with $2 \odot 4$ or $\odot 6$ as compared with $\odot 4$, in the strain of $\odot 8$ as compared with $2 \odot 4$ or $\odot 6$, and further in $\odot 12$ as compared with $\odot 8+2_{\text {II }}$ (table 2). The strains with $\odot 1,2 \odot 6$ and $\odot 12$ were all completely pollen sterile, because the anthers of these strains were shrunken and did not dehisce.

TABLE 2

Pollen fertility (percentage) in original and $X$-ray-induced reciprocal translocation strains

$\begin{array}{cccccc}6 \text { II } & \odot 4+4_{\mathrm{II}} & \odot 6+3_{\mathrm{II}} & 2 \odot 4+2_{\mathrm{II}} & \odot 8+2_{\mathrm{II}} & \odot 1^{*} \\ 90.3 & 70.5 & 52.8 & 53.3 & 29.3 & 0.0\end{array}$

* $2 \odot 1+5_{\mathrm{II}}, \odot 4+2 \odot 1+3_{\mathrm{II}}$ and $\odot 4+\odot 3+\odot 1+2_{\mathrm{II}}$ inclusive. 

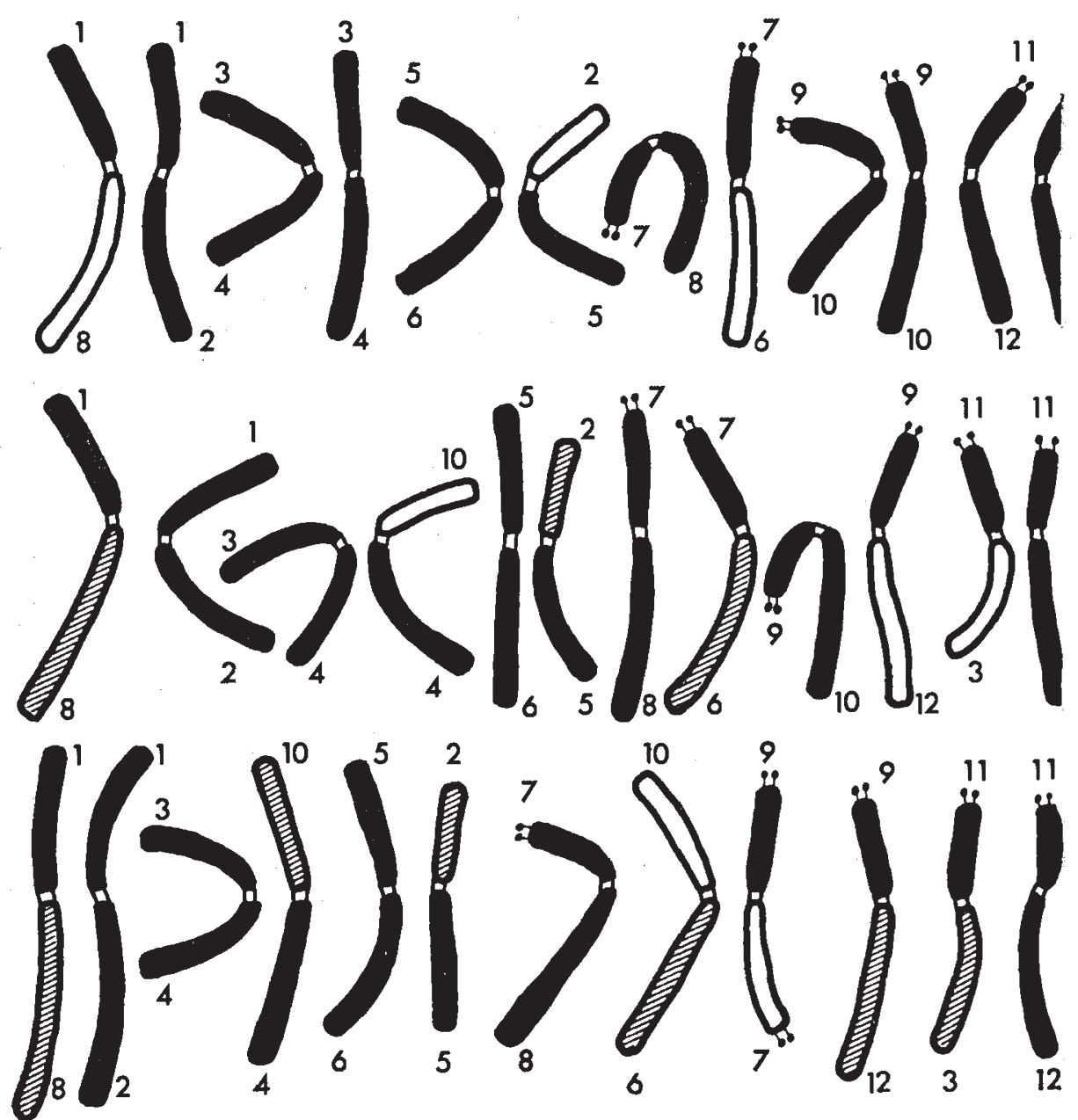

Fras. 20-22.-Chromosome sets in root-tip cells showing three steps of changing karyotypes accompanied by successive translocations from $6_{\mathrm{II}}$ to $\odot 12$. Translocated arms in each step are indicated in blank and translocated arms of the foregoing translocations in oblique line. $\times 2000$.

Frg. 20.- $\odot 6+3$ II, primary strain from the standard (fig. 15).

Frc. $21 .-2 \odot 6$, secondary strain from the primary $\odot 6+3_{\mathrm{II}}$ (fig. 20).

Fig. 22.- $\odot 12$, tertiary strain from the secondary $2 \odot 6$ (fig. 21).

Complete pollen sterility of the strains involving $\odot 1$ is due to a geneticphysiologic disturbance caused by genetic duplications and/or deficiencies which depend on AI distribution of $\odot 1$ chromosomes, for the two arms of pseudo-isochromosomes are genetically nearly equal except for a short region adjacent to centromere. Complete pollen sterility in the strains involving $2 \odot 6$ or $\odot 12$ is also caused by duplications and/or deficiencies resulting from non-disjunction of the ring(s). Simply based on the finding that approximate decrease in pollen fertility is about 20 per cent. per each one reciprocal translocation, good pollen fertility is not to be expected in these strains. Seed setting is poor even in the mother strain $6 \mathrm{II}$, at least in 
Japan. The strains of $\odot 4$ set few seeds, but the strains with many and/or large rings are completely sterile.

\section{Discussion}

On the assumption that breakages and reunions occur at random, a formula $4(\mathcal{N}-1) / 1$ for calculating the expected ratio of rings and pseudoisochromosomes was presented by Koo (1959), where $\mathcal{N}$ stands for the number of chromosome pairs. According to his formula, the expected ratio of rings to pseudo-isochromosomes in Tradescantia paludosa $(2 n=12)$ is expected as 20/1. In the primary reciprocal translocation strains, the number of reciprocal translocations resulting in rings is 48 and those resulting in pseudo-isochromosomes 4 (table 1 ). The $\chi^{2}$-test for these estimates showed $0.50>\mathrm{P}>0.30\left(\chi^{2}=0.607\right.$, d.f. $\left.=1\right)$, i.e. reciprocal translocations had happened at random between chromosomes irrespective of whether they are homologous or non-homologous.

The total number of interchanged and intact chromosomes in five types of reciprocal translocations in primary strains was 32 and 76 respectively, i.e. 2 and 10 in $\odot 4+4_{\mathrm{II}}, 3$ and 9 in $\odot 6+3_{\mathrm{II}}, 4$ and 8 in $2 \odot 4+2_{\mathrm{II}}, 4$ and 8 in $\odot 4+2 \odot 1+3$ II and 5 and 7 in $\odot 4+\odot 3+\odot 1+2$ II. On the other hand, the total number of interchanged and intact chromosomes participating in the secondary reciprocal translocations is calculated as $5 \frac{1}{6}$ and $14 \frac{5}{6}$ respectively. These numbers were obtained as follows. For example, secondary $2 \odot 4+2$ II from primarily $\odot 4+4$ II resulted from a secondary reciprocal translocation between two primary unaffected chromosomes, i.e. 2 intact and no interchanged chromosomes participated in the secondary translocation. But, $\odot 6+3$ II from $\odot 4+4$ II results from two breaks, one in a primarily intact chromosome and another one may take place in intact or interchanged chromosomes with equal chance. Accordingly, in this case the chromosomes participating in the secondary reciprocal translocation are calculated as $1+\frac{1}{2}$ for intact and $\frac{1}{2}$ for interchanged chromosomes. In addition, $\odot 5+\odot 4+\odot 1+1_{\text {II }}$ from $\odot 4+\odot 3+\odot 1+2_{\text {II }}$ also resulted from two breaks, one in a primarily intact chromosome and another one may take place in an intact or interchanged chromosome involved in $\odot 3$ with equal chance. Therefore, in this case, the calculated values are presented as $1+\frac{1}{3}$ for intact and $\frac{2}{3}$ for interchanged chromosomes. Totals of the values calculated in the same way as mentioned above for all nine secondary strains were $4+\frac{1}{2}+\frac{2}{8}$ and $14+\frac{1}{2}+\frac{1}{3}$, i.e. $5 \frac{1}{6}$ and $14 \frac{5}{6}$.

If breaks by the second irradiation take place at random without distinction between primarily intact and interchanged chromosomes, the ratio $32: 76(5 \cdot 9: 14.1)$ calculated in the foregoing lines for the primary strains will be maintained again in the secondary strains. No significant differences were found between the expected ratio 5.9:14.1 and the observed ratio $5 \frac{1}{6}: 14 \frac{5}{6}(5 \cdot 2: 14 \cdot 8)\left(\chi^{2}=0.166\right.$, d.f. $\left.=1,0.70>P>0.50\right)$, indicating that breaks by the second irradiation occur at random irrespective of intact or interchanged chromosomes.

In the considerations mentioned above as well as in the following considerations, only two breaks, one each in different chromosomes, were assumed in order to simplify the deduction, though it might be an oversimplification.

Four arm combinations are possible in reunion of two breaks, one each in different chromosomes, but pseudo-isochromosomes result from only two. 
combinations in every homologous pair. In the cases where the two chromosomes are non-homologous a ring-of-four results from every one out of four combinations. Consequently, $2\left(\begin{array}{c}12 \\ 2\end{array}\right)=132$ arm combinations are possible in Tradescantia paludosa. An analysis of these 132 possible arm combinations inducible by further irradiation to $\odot 4+4$ II revealed that alterations classifiable into six types of catenations occur (table 3 ).

An additional reciprocal translocation between two chromosomes of the members consisting of $\odot 4$ produces three types catenations: $\odot 4+4$ II, $\odot 3+\odot 1+4$ II and 6 II. The reciprocal translocation between one of the members of $\odot 4$ and one of the members of 4 II produces consistently $\odot 6+3_{\text {II }}$.

TABLE 3

Types and their frequencies of secondary types expected from irradiation to $\odot 4+4$ II

\begin{tabular}{|c|c|c|}
\hline Secondary types & Expecte & quencies \\
\hline $\begin{array}{l}\odot 6+3 \mathrm{II} \\
2 \odot 4+2 \mathrm{II} \\
\odot 4+2 \odot 1+3 \mathrm{II} \\
\odot 4+4 \mathrm{II} \\
\odot 3+\odot 1+4 \mathrm{II} \\
6_{\mathrm{II}}\end{array}$ & $\left.\begin{array}{r}64 \\
48 \\
4\end{array}\right\} 116$ & $\begin{array}{c}(87.9 \%) \\
(7.6 \%) \\
(4.5 \%)\end{array}$ \\
\hline Total & 32 & $\%$ \\
\hline
\end{tabular}

TABLE 4

Types and their frequencies of secondary types expected from irradiation to $\odot 6+3_{\text {II }}$

Secondary types

Expected frequencies

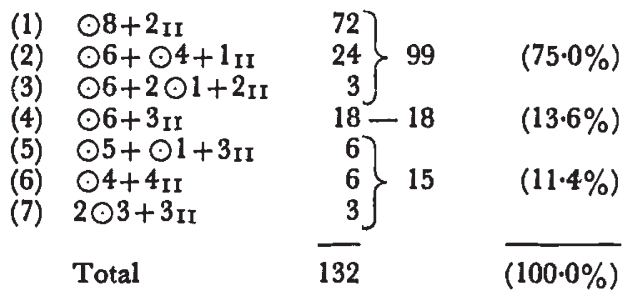

However, a reciprocal translocation between the members of $4_{\text {II }}$ results in $2 \odot 4+2 I I, \odot 4+2 \odot 1+3 I$ and $\odot 4+4 I I$. Catenations of type (1), (2) and (3) in the table are more advanced than the original $\odot 4+4$ II, relative frequency amounting to 87.9 per cent. of all the cases expected. Type (4) is the same as the original, its frequency being $7 \cdot 6$ per cent. In types (5) and (6) catenations are reduced from the original one. The total frequency of types (5) and (6), 4.5 per cent., would be reduced to 1.5 per cent. if type (5) is excluded as a case of reversion from $\odot 4$ to $2_{I I}$.

The same analyses as in the foregoing case on results from $\odot 6+3_{\text {II }}$ and $2 \odot 4+2$ II also revealed less frequent reduction in catenations (tables 4 and 5). In the strain with the largest ring, $\odot 12$, only one-half of the possible resultants remains in the type of catenation $\odot 12$, and the other half is of the types of catenation reduced from the original $\odot 12$ (table 6 ). However, these types have much less possibility of reduction in catenation by the next or successive interchange. 
Throughout the cases mentioned above breakdown of rings by an additional interchange is much less frequent than addition of further chromosomes into the ring(s), i.e. the successive occurrence of interchanges strongly tends to increase the size of rings. Indeed, in the present experimental study all of the secondarily induced reciprocal translocation strains showed an increase in the number of chromosomes in catenations.

TABLE 5

Types and their frequencies of secondary types expected from irradiation to $2 \odot 4+2 \mathrm{II}$

\section{Secondary types}

(1) $\odot 8+2$ II

(2) $\odot 6+\odot 4+1_{\text {II }}$

(3) $3 \odot 4$

(4) $2 \odot 4+2 \odot 1+1 \mathrm{II}$

(5) $2 \odot 4+4$ II

(6) $\odot 4+\odot 3+\odot 1+2$ II

(7) $\odot 4+4$ II

Total
Expected frequencies

32

$64\} 106$

$8\}$

$14-14$

4\} 12

132
$(80 \cdot 3 \%)$

$(100 \cdot 0 \%)$

TABLE 6

Types and their frequencies of secondary types expected from irradiation to $\odot 12$

Secondary types

Expected frequencies
(1) $\odot 12$
(2) $\odot 11+\odot 1$
(3) $\odot 10+1_{\text {II }}$
(4) $\odot 9+\odot 3$
(5) $\odot 8+\odot 4$
(6) $\odot 7+\odot 5$
(7) $2 \odot 6$

Total

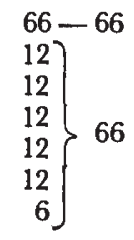

132
$(50.0 \%)$

$(50 \cdot 0 \%)$

$(100.0 \%)$

Thus, in longlived perennial plants reciprocal translocations can be accumulated without combining independent translocations by interbreeding. The accumulation in this way will finish at last by achieving the strain(s) with the ultimate largest ring-of- $2 n$ chromosomes.

Acknowledgments. - The author wishes to express his gratitude to Professor T. Haga, for his invaluable advice and criticism. The author is indebted to $\mathrm{Dr} \mathrm{H}$. Kihara, the former President of the National Institute of Genetics, for his supplying the materials, which introduced by him to Japan in 1948 from Bussey Institution, Harvard University, the United States. He is also grateful to Dr Ruby I. Larson, Canada Department of Agriculture, who kindly polished up the manuscript.

\section{REFERENCES}

BURnham, C. R. 1956. Chromosomal interchanges in plants. Bot. Rev., 22, 419-552. CALDECOTT, R. S., AND sMITH, L. 1952. A study of X-ray induced chromosomal aberrations in barley. Cytologia, 17, 224-242.

Cleland, R. E. 1962. The cytogenetics of Oenothera. Adv. Genet., 11, 147-237.

DARLINGTON, C. D. 1929. Chromosome behaviour and structural hybridity in the Tradescantiae. F. Genet., 21, 207-286.

Darlington, C. D. 1931. The cytological theory of inheritance in Oenothera. F. Genet., 24, 405-474. 
Koo, F. K. s. 1959. Expectations on random occurrence of structural interchanges between homologous and between non-homologous chromosomes. Amer. Naturalist, 93, 193-199. LAWRENCE, c. w. 1958. Genotypic control of chromosome behaviour in rye. VI. Selection for disjunction frequency. Heredity, 12, 127-131.

NEWCOMER, E. H. 1953. A new cytological and histological fixing fluid. Science, 118, 161.

THOMPson, J. B. 1956. Genotypic control of chromosome behaviour in rye. II. Disjunction at meiosis in interchange heterozygotes. Heredity, 10, 99-108.

watanabe, H. 1959. X-ray induced reciprocal translocations in Tradescantia paludosa. Jap. 7. Genet., 34, 162-167.

Watanabe, H. 1962a. An X-ray-induced strain of ring-of-12 in Tradescantia paludosa. Nature, 193, 603.

WATANABE, H. 1962b. A short-cut in producing a strain of ring-of-12 by X-rays in Tradescantia paludosa. Chromosome Information Service, 3, 48.

YAMASHrTA, K. 1951. Studies on X-ray induced reciprocal translocations in Einkorn wheats. III. A newly synthesized ring of 14 chromosomes in a complex heterozygotes, aegilopoides-monococcum. Cytologia, 16, 164-176.

YAMAsHTTA, K. 1952. Studies on X-ray induced reciprocal translocations in Einkorn wheats: II. Rep. Kihara Inst. Res. (Seiken Zihô), 5, 1-41.

YAMASHTTA, K. 1953. Studies on X-ray induced reciprocal translocations in Einkorn wheats: V. Jap. J. Genet., 28, 238-247. 